\title{
Properties and origin of energetic particles at the duskside of the Earth's magnetosheath throughout a great storm
}

\author{
D. V. Sarafopoulos ${ }^{1}$, M. A. Athanasiu ${ }^{1}$, E. T. Sarris ${ }^{1}$, T. Yamamoto ${ }^{2}$, S. Kokubun ${ }^{3}$ \\ ${ }^{1}$ Demokritos University of Thrace, Department of Electrical and Computer Engineering, Xanthi, GR-67100, Greece \\ ${ }^{2}$ Institute of Space and Astronautical Science, 3-1-1 Yoshinodai, Sagamihara 229, Japan \\ ${ }^{3}$ Solar-Terrestrial Environment Laboratory, Nagoya University, Toyokawa 442, Japan
}

Received: 26 August 1998 / Revised: 27 January 1999 / Accepted: 5 February 1999

\begin{abstract}
We study an interval of 56 h on January 16 to 18, 1995, during which the GEOTAIL spacecraft traversed the duskside magnetosheath from $X \cong-15$ to $-40 R_{E}$ and the EPIC/ICS and EPIC/STICS sensors sporadically detected tens of energetic particle bursts. This interval coincides with the expansion and growth of a great geomagnetic storm. The flux bursts are strongly dependent on the magnetic field orientation. They switch on whenever the $B_{z}$ component approaches zero $\left(B_{z} \cong 0 \mathrm{nT}\right)$. We strongly suggest a magnetospheric origin for the energetic ions and electrons streaming along these "exodus channels". The time profiles for energetic protons and "tracer" $\mathrm{O}^{+}$ions are nearly identical, which suggests a common source. We suggest that the particles leak out of the magnetosphere all the time and that when the magnetosheath magnetic field connects the spacecraft to the magnetotail, they stream away to be observed by the GEOTAIL sensors. The energetic electron fluxes are not observed as commonly as the ions, indicating that their source is more limited in extent. In one case study the magnetosheath magnetic field lines are draped around the magnetopause within the $\mathrm{YZ}$ plane and a dispersed structure for peak fluxes of different species is detected and interpreted as evidence for energetic electrons leaking out from the dawn LLBL and then being channelled along the draped magnetic field lines over the magnetopause. Protons leak from the equatorial dusk LLBL and this spatial differentiation between electron and proton sources results in the observed dispersion. A gradient of energetic proton intensities toward the $\mathrm{Z}_{\mathrm{GSM}}=0$ plane is inferred. There is a permanent layer of energetic particles adjacent to the magnetosheath during this interval in which the dominant component of the magnetic field was $B_{z}$.
\end{abstract}

Key words. Magnetospheric physics (magnetosheath; magnetotail boundary layers; storms and substorms)

Correspondence to: D. V. Sarafopoulos

\section{Introduction}

This work concerns the key-role of the $B_{z}$ component of the magnetic field in the detection of energetic burst-like fluxes of magnetospheric origin within the duskside magnetosheath. Exodus of energetic particles out of the magnetospheric cavity takes place only via distinct channels along the magnetic field lines, particularly from 15 to $40 R_{E}$ downstream. The magnetosheath region is divided into a permanent layer of particles adjacent to the magnetopause and ephemeral channels of particles streaming away from the magnetopause. We identify features favouring leakage through the magnetopause and show a very good association between the energetic $\mathrm{O}^{+}$and $\mathrm{H}^{+}$time profiles for the first time in the nightside magnetosheath. In general, a deeper understanding of the energetic particles properties within the magnetosheath is gained.

The magnetosheath as an "autonomous region" has received less attention than the other parts of geospace and this is especially true for the nightside magnetosheath. Hones et al. (1972) reported the existence of 10 to $30 \mathrm{keV}$ protons in the magnetosheath plasma and suggested the magnetosphere as the source of this population. The first comprehensive survey of $\geq 100 \mathrm{keV}$ ions in the magnetosheath is that reported by West and Buck (1976). They found that the energetic ion fluxes correlated with both geomagnetic activity and enhanced turbulence in the magnetosheath magnetic field. Detailed correlations of proton fluxes with particular substorm phases were unsuccessful. They observed power law ion spectra in the magnetosheath near the magnetopause quite similar to those in the nearby magnetosphere. The source of $\geq 100 \mathrm{keV}$ magnetosheath ions could be the magnetosphere and/or the energization of low-energy protons in the magnetosheath, the bow shock, or the upstream wave region. Sarris et al. (1976) found that energetic $\left(E_{p} \geq 290 \mathrm{keV} ; E_{e} \geq 220 \mathrm{keV}\right)$ proton and electron bursts are a semi-permanent feature of 
the near-Earth environment both within and outside the magnetotail and concluded that the energetic magnetosheath ions are of a nonthermal origin. Sarris et al. (1978) presented simultaneous observations from three spacecraft and argued that proton bursts occur nearly simultaneously inside the magnetosphere, in the magnetosheath, and upstream from the bow shock. They suggested that energetic protons and electrons most likely are accelerated inside the plasma sheet and then propagate to various regions. Asbridge et al. (1978) reported that 3 to $40 \mathrm{keV}$ ions are present sporadically in the magnetosheath and proposed they are produced in the vicinity of the bow shock. Williams (1979) presented strong evidence for significant but sporadic magnetosheath energy flow in the antisolar direction using 50 $220 \mathrm{keV}$ ions. Williams et al. (1988) studied two magnetosheath traversals, one on each of the dawn and dusk flanks of the magnetosphere. They concluded that the magnetosheath plasma often has at least two major components, the shocked solar wind seen at energies $\leq 5 \mathrm{keV}$ (which behaves independently of the magnetic field direction) and the magnetospheric (plasma sheet) particles seen at energies $\geq 5 \mathrm{keV}$ (which are highly modulated by the magnetic field). Also they found that, at times, the magnetosheath plasma possibly consisted only of the shocked solar wind, with the observed highenergy tail perhaps due to ambient energetic solar ions or acceleration processes at the bow shock. In one case study, at the dawn magnetosheath $\sim 50 R_{E}$ downstream, Scholer et al. (1984) observed, with the ISEE-3 spacecraft, electron and proton bursts occurring almost simultaneously. In general, it is worth noticing that although streaming fluxes of energetic particles are extensively studied in the dayside magnetosheath (see Table 1 in Sibeck et al., 1987a), corresponding work for the nightside magnetosheath is missing.

In this article with fine time-resolution data-sets, we establish the phenomenon of the exodus of magnetospheric energetic population via distinct channels. We determine their morphology and properties and proceed further in order to monitor processes over the magnetopause using their microstructure.

\section{Observations and data analysis}

\subsection{Spacecraft locations and instrumentation}

The period to be examined closely is the interval from the beginning of day 16,1995 , to 08:00 UT on 18 January, 1995, (i.e. $56 \mathrm{~h}$ in all). During this interval the GEOTAIL spacecraft traversed the dusk magnetosheath from $\mathrm{X}=-14.5$ to $-39.9 R_{E}$ and terminated its trajectory by repeatedly crossing the magnetopause boundary. Projections of the spacecraft positions on the $\mathrm{YZ}_{\mathrm{GSE}}$ and $\mathrm{XY} \mathrm{Y}_{\mathrm{GSE}}$ planes are given in Fig. 1a, b. Also shown for reference are the nominal magnetopause and bow shock positions for average solar wind conditions. It is seen on the $\mathrm{YZ}_{\mathrm{GSE}}$ plane that the spacecraft gradually shifted from $\mathrm{Z}_{\mathrm{GSE}}=-3.4$ to -6.4 $R_{E}$ during the interval. The latter leads to the inference that the GEOTAIL spacecraft essentially remained close to the ecliptic plane.

The identification of different plasma regimes was made with plasma parameters obtained via the Solar Wind and Hot Plasma Analyzers (SWA and HPA respectively) of the Comprehensive Plasma Instrument (CPI, see Frank et al., 1994). These data are shown in the GSE system of coordinates with 48 s resolution. The CPI consists of three plasma analyzers: (1) an electrostatic analyzer for hot electrons and ions (HPA), (2) a complementary electrostatic analyzer for cool plasmas (SWA) with high bulk speeds such as those found within the solar wind and magnetosheath, and (3) an ion composition analyzer for identification of ion species such as $\mathrm{H}^{+}, \mathrm{He}^{+}, \mathrm{He}^{++}$, and $\mathrm{O}^{+}$. The energy-per-unit charge ranges for these analyzers are $1.3 \mathrm{~V}$ to $48.2 \mathrm{kV}, 145 \mathrm{~V}$ to $6830 \mathrm{~V}$, and $1.3 \mathrm{~V}$ to $48.2 \mathrm{kV}$, respectively. For the present analysis we utilize magnetic field data from the MGF experiment (see Kokubum et al., 1994) with 3 s resolution in the GSM system. The $B_{z}$ component of the IMF is given via the MFI experiment on board the WIND spacecraft (see

a
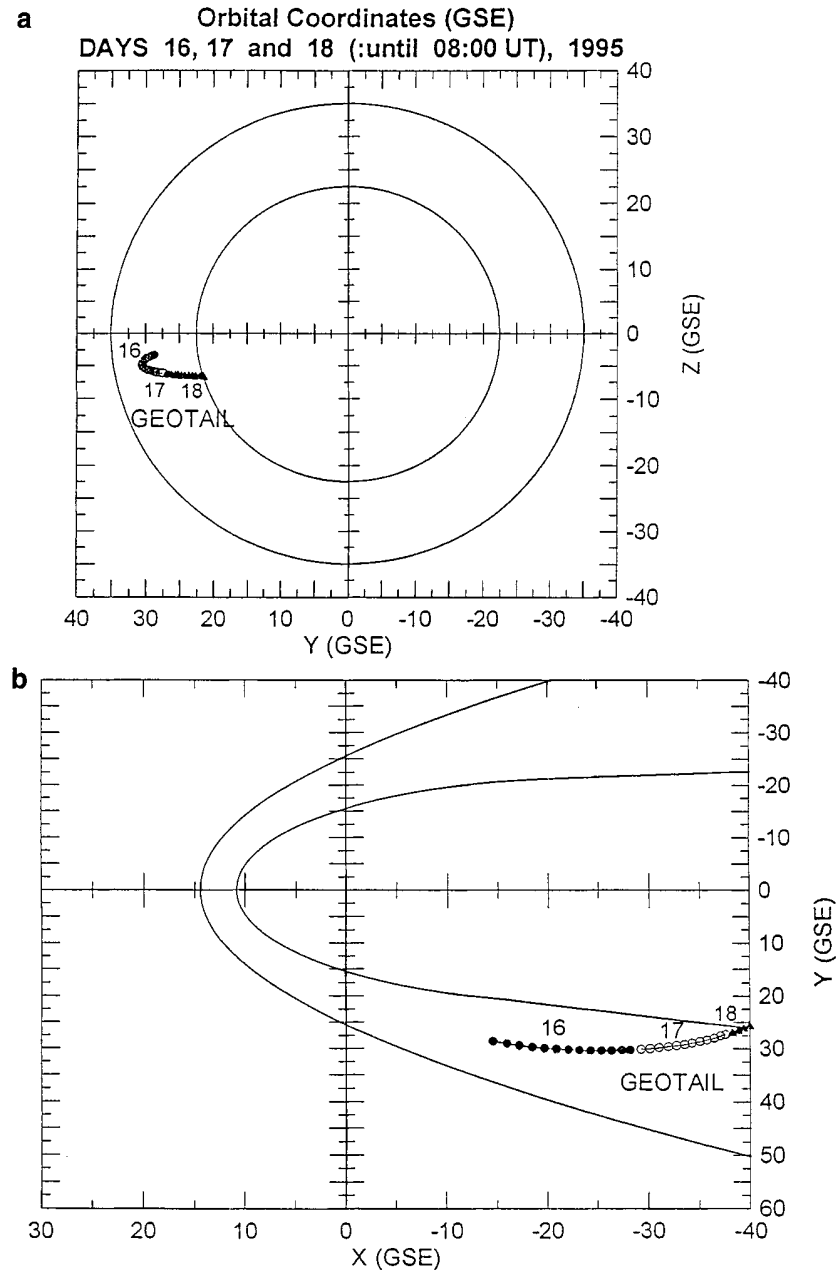

Fig. 1a, b. GEOTAIL trajectories projected on the $\mathrm{YZ}_{\mathrm{GSE}}$ and $\mathrm{XY}_{\mathrm{GSE}}$ planes and corresponding to the days 16 (solid circles), 17 (open circles) and 18 (until 08:00 UT, solid triangles), 1995. Average bow shock and magnetopause positions are also shown 
Lepping et al., 1995). The energetic particle differential fluxes are provided via the Energetic Particles and Ion Composition (EPIC) instrument (see Williams et al., 1994). EPIC consists of sensors ICS and STICS, while the time resolution is declared in each plot varying from $3 \mathrm{~s}$ to $96 \mathrm{~s}$. The STICS sensor provides $\sim 4 \pi$ angular coverage, composition and spectral observations, with charge state determination for all ions from $30 \mathrm{keV}$ to $230 \mathrm{keV} / \mathrm{e}$, and mass per charge measurements $\geq 7.5 \mathrm{keV} / \mathrm{e}$. The ICS sensor provides flux, composition, spectra, and angular distributions over two polar angles of the elemental species protons through iron from $\geq 50 \mathrm{keV}$ to $3 \mathrm{MeV}$ along with angular distributions in one plane of electron fluxes $\geq 38 \mathrm{keV}$ and $\geq 110 \mathrm{keV}$. The two polar planes correspond to two nearly identical ion heads (north and south) with conical collimators, where the north (south) is centred $23^{\circ}$ degrees above (below) the ecliptic plane. The "head 0 " corresponds to the north collimator.

\subsection{Region identification and geomagnetic activity}

The identification of the different plasma regimes traversed by the GEOTAIL spacecraft has been made on the basis of the CPI experiment. In Fig. 2 are displayed from the SWA the density (in $\mathrm{cm}^{-3}$, top panel), the three components of ion velocity $\mathrm{V}_{\mathrm{x}}, \mathrm{V}_{\mathrm{y}}$ and $\mathrm{V}_{\mathrm{z}}$ (in $\mathrm{kms}^{-1}$, third, fourth and fifth panels respectively), as well as the ion average energy (in $\mathrm{eV}$, second panel) from the HPA. The high levels of density and velocity of the tailward ion fluxes ensure that the spacecraft remains inside the magnetosheath. In contrast, after about 05 UT of day 18, 1995, at times, the very low ion SWA densities, as well as the dramatically enhanced kinetic temperatures of the HPA, indicate recurrent magnetopause crossings.

The whole time interval we are interested in is associated with the main phase of a magnetic storm. The sixth panel of Fig. 2 displays the $D_{s t}$ index with a minimum value of $-95 \mathrm{nT}$ giving evidence about a sufficiently intensified ring current almost reaching the bottom threshold of $-100 \mathrm{nT}$ designated for a great storm (Gonzalez et al., 1994). The $B_{z}$ component of the IMF, as recorded on board the WIND spacecraft at (X, $\mathrm{Y}, \mathrm{Z})_{\mathrm{GSE}} \cong(150,-75,-10) R_{E}$, is shown in the bottom panel of Fig. 2. It is noticed that the $-B_{z}$ component exceeds the threshold of $-10 \mathrm{nT}$ for an interval of $\sim 9 \mathrm{~h}$, leading us to classify the storm considered as clearly falling into the great category (see Gonzalez and Tsurutani, 1987).

\subsection{An interval with recurrent "exodus channels"}

There were numerous energetic particle bursts in the magnetosheath throughout the whole interval. We are focusing only on a few of them. Figure 3 is a first example, where the three components, as well as the magnitude of the magnetic field ( $3 \mathrm{~s}$ resolution), are shown along with the differential fluxes of the 58$77 \mathrm{keV}$ energetic protons (6 s resolution). The fluxes

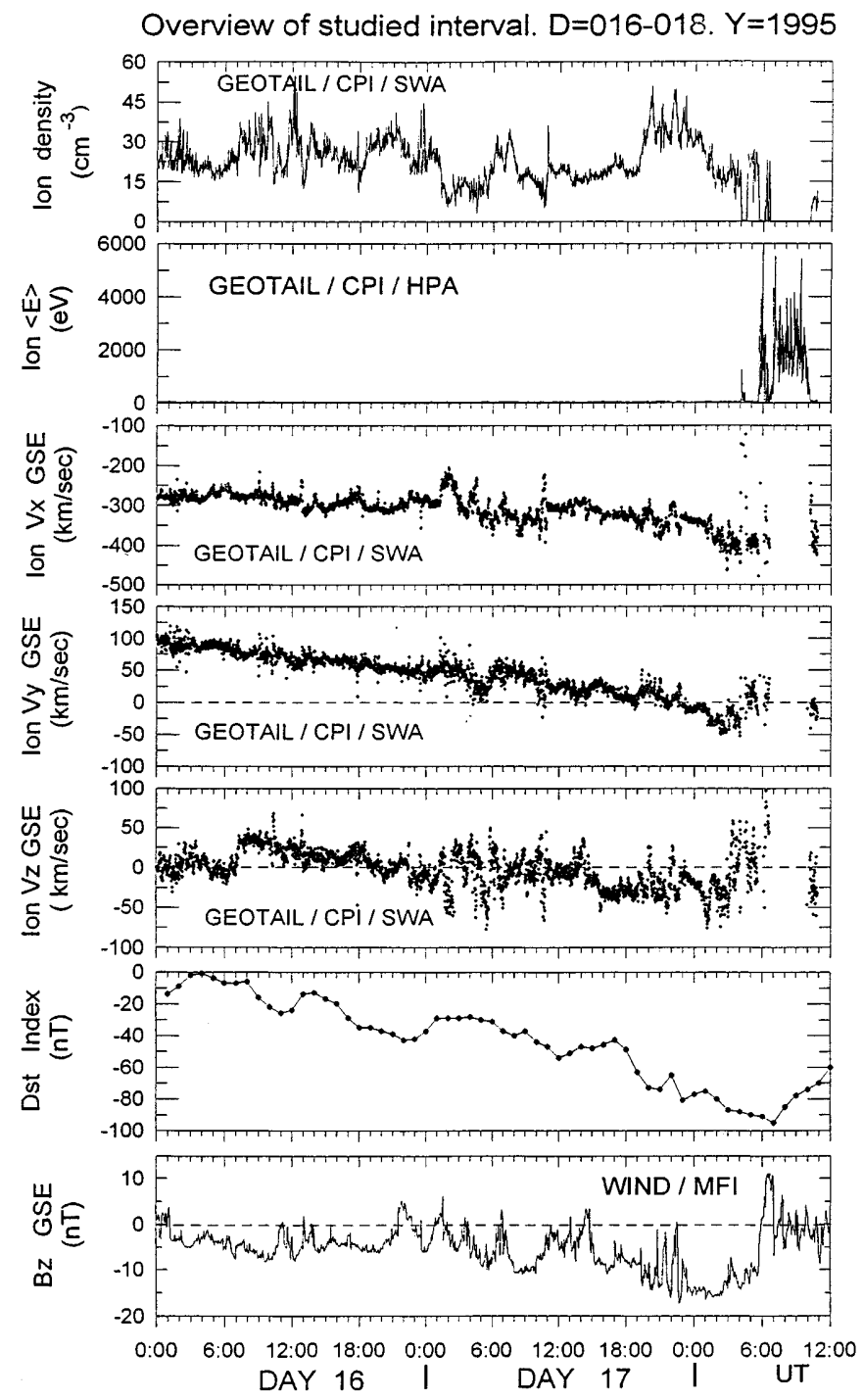

Fig. 2. Comprehensive Plasma Instrument (CPI) data are used to identify the spacecraft traversed regions. From top to bottom the ion density and average energy density, the three components of plasma velocity, the ground-based $D s t$ index and the IMF $B_{z}$ component (as recorded onboard WIND via the MFI experiment) are shown. The density and velocity have measured by the Solar Wind Analyser, while the average ion energy by the Hot Plasma Analyser

are spin $(\sim 3 \mathrm{~s})$ averaged. The vertical dashed lines and the marked arrows emphasize the $B_{z}$ and peak flux association. In general, with dominant $B_{z}$ component, the magnetic field structure is devoid of energetic particles, whereas with near zero $B_{z}$ massive exodus of energetic particle population takes place via the opened corridor of the magnetic tube. Whenever the $B_{z}$ approaches zero, it seems that a magnetic connection is established between the spacecraft and the magnetotail. For instance, at $\sim 01: 32$ UT of day 16, 1995, a burst peaked at the time when the $B_{z}$ changes sign.

At this point, before the presentation of additional cases of exodus channels, it is important to look at the burst-like fluxes in connection with the CPI data. Throughout the interval studied, the GEOTAIL space- 


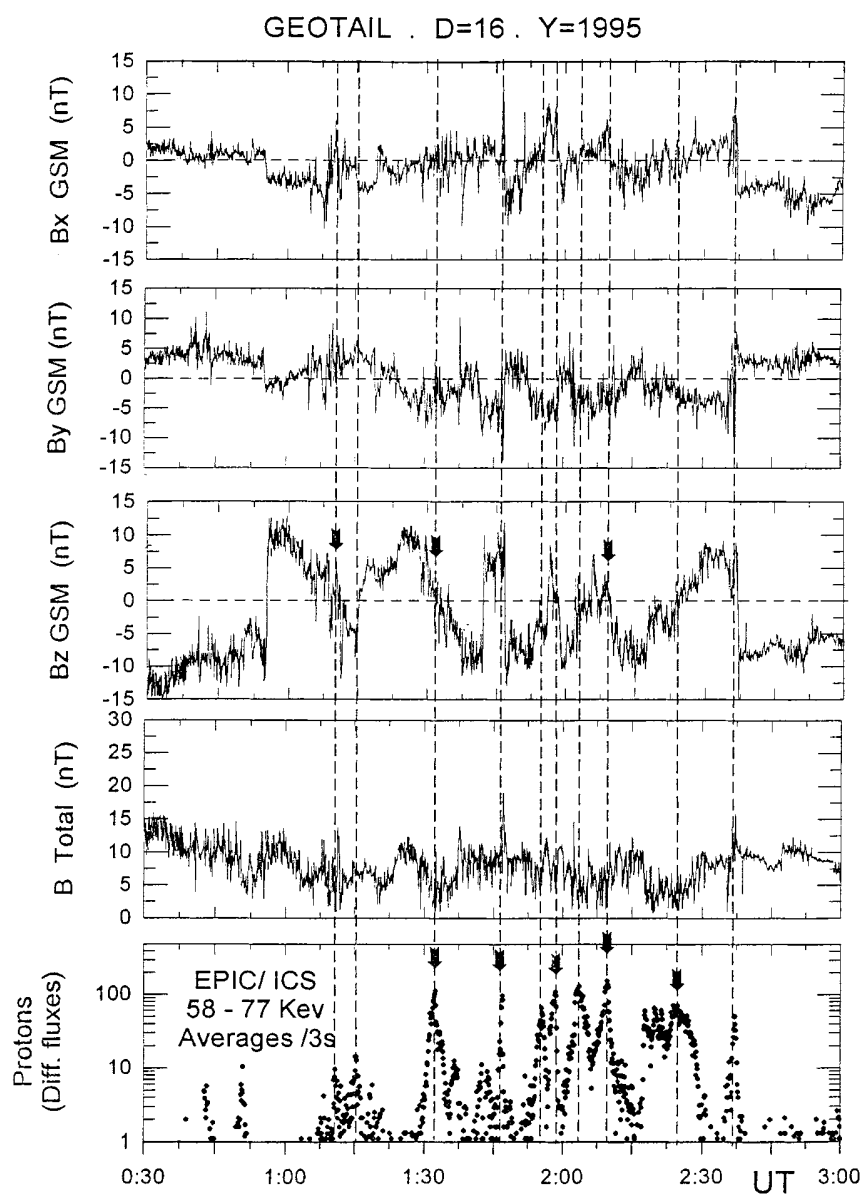

Fig. 3. Vector magnetic field measurements with $3 \mathrm{~s}$ resolution from the MGF experiment in GSM system of coordinates, along with the EPIC/ICS 58-77 keV energetic proton fluxes for the period 00:30 03:00 UT of day 16, 1995. About ten successive bursts of energetic protons occurred whenever $B_{z} \cong 0 \mathrm{nT}$. The arrows and dashed lines show the times when the fluxes peaked along the $B_{z}$ trace

craft is actually moving parallel to the model magnetopause. Thus, it is conceivable to explain the GEOTAIL observations in terms of a series of short-term encounters with a fluctuating magnetopause, i.e. the periods with energetic particle bursts and magnetic field discontinuities may correspond to such sporadic encounters. In order to analyze this possibility we examine distinct burst examples with the accompanying high-resolution plasma data from the SWA instrument. One particular case is shown in Fig. 4, where the intense flux of energetic protons is shown in the bottom panel. The magnetic field measurements (upper panels) are shown using the same format as in Fig. 3. The plasma density (fifth panel) and velocity (sixth panel), as well as the flow direction remain essentially undisturbed revealing a core magnetosheath plasma. Phi $(\phi)$ and theta $(\vartheta)$ are the azimuthal and polar angles (in degrees) of the plasma flow. There is no indication in the plasma data for intermittently approaching the magnetopause. Consequently, we conclude that these particle bursts are related only to prominent magnetic field changes and not to magnetopause motion.

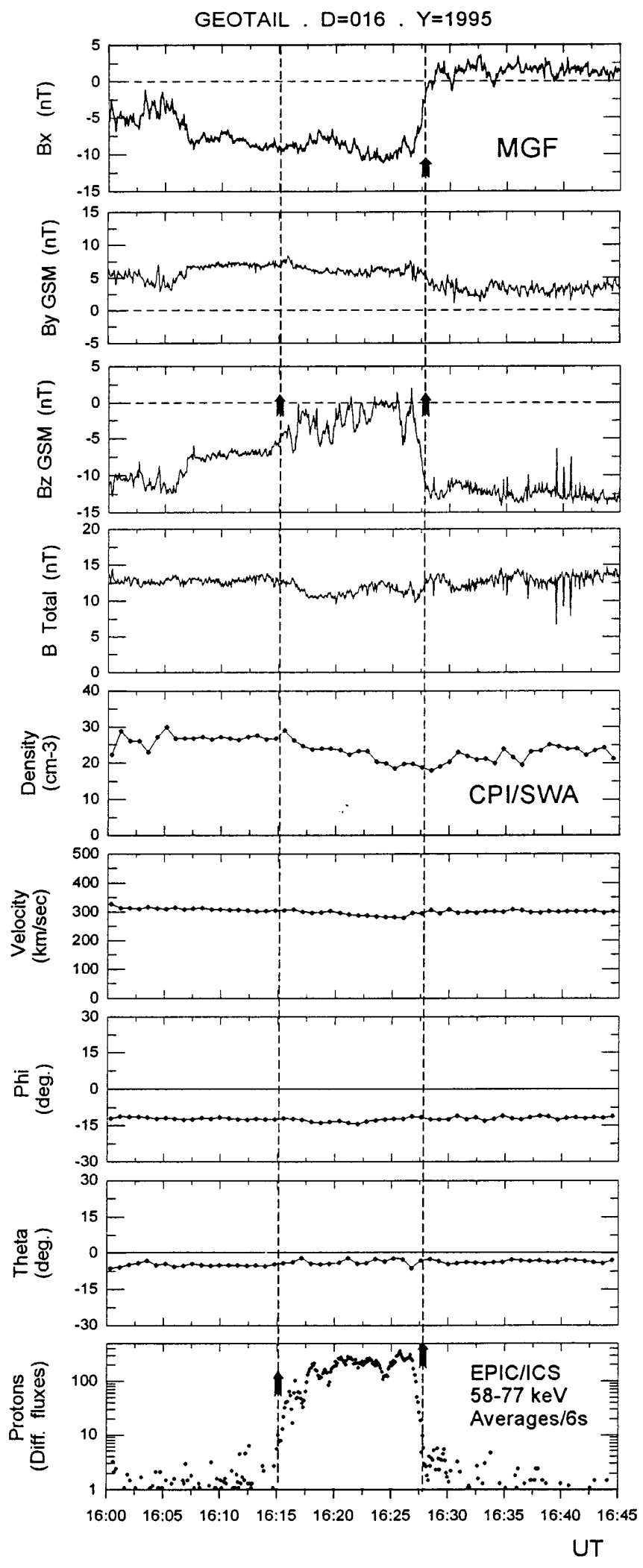

Fig. 4. Vector magnetic field measurements and the highest resolution available data from the CPI/SWA experiment for 16:00-16:45 UT of day 16, 1995. The energetic protons burst is shown in the bottom panel. The plasma density and velocity (that is, the magnitude, azimuth $\phi$ and latitude $\vartheta$ angles) do not show any significant burstassociated variations 


\subsection{A distinct burst with velocity dispersion structure}

During the interval from 01:25 to 01:40 UT of day 16, 1995, the GEOTAIL spacecraft was located at (X, Y, $\mathrm{Z})_{\mathrm{GSE}} \cong(-15.9,29.1,-3.6) R_{E}$. The top panel of Fig. 5 displays the magnitude of the magnetic field (thick line), as well as the $B_{z}$ component, while the second and third panels show the azimuth $\varphi$ and latitude $\vartheta$ angles. The direction of zero $\phi$ and $\vartheta$ points sunward, while the directions of $90^{\circ}$ for $\phi$ and $\vartheta$ point duskward and northward, respectively. It is evident that the vector magnetic field scans gradually all the directions from northward to southward. Most importantly, the interval of near zero $B_{z}$ is accompanied with energetic 58-77 keV proton (fourth panel), 187-222 keV CNO ion (fifth panel) and $\geq 38 \mathrm{keV}$ electron (bottom panel) fluxes. When the energetic electron fluxes are peaked

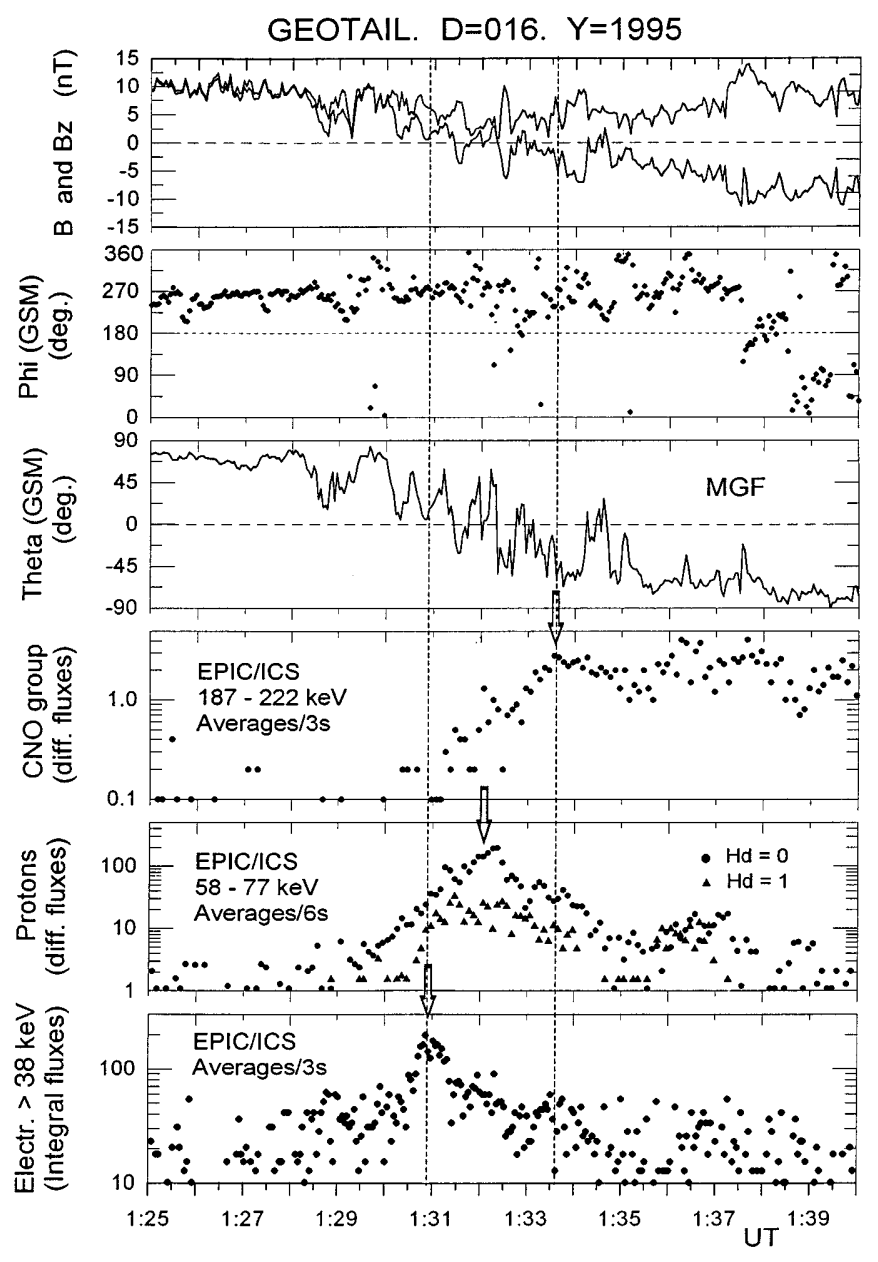

Fig. 5. A distinct burst of energetic particles (bottom three panels) along with magnetic field data (upper three panels) are shown for the short period 01:25-01:40 UT of day 16, 1995. The upper panel shows the magnetic field magnitude as well as the $B_{z}$ component (thinner line). The azimuthal $\varphi$ and latitude $\theta$ angles are displayed in second and third panels. The three arrows mark the times of peaks for the energetic 58-77 keV protons (fourth panel), 187-222 keV CNO ions (fifth panel) and $>38 \mathrm{keV}$ electron fluxes (bottom panel) demonstrating a species dependent dispersion structure. Around the peak fluxes the $B_{z}$ component gradually changes sign the vector of the magnetic field essentially lies on the YZ plane. The maximum of energetic electrons essentially precedes the peak fluxes of protons. The electron fluxes reach a peak $\sim 150 \mathrm{~s}$ prior to the CNO ions. The constructed average angular distributions of the peak fluxes for the electrons $(\geq 38 \mathrm{keV})$, protons $(58-77 \mathrm{keV})$ and the CNO group ions (187-222 keV) are shown in Fig. $6 \mathrm{a}, \mathrm{b}$ and $\mathrm{c}$, respectively. The electron fluxes are mainly directed duskward parallel to the average magnetic field direction. The same is valid for the observed proton fluxes which show distinct fieldaligned streaming. The heavy $\mathrm{CNO}$ ions show a dominant antisunward convective component in the direction of the plasma flow in the duskward magnetosheath. An interpretation for the observed dispersion is given at the discussion section.

\subsection{The electron channels are rare compared to those of proton}

This case study, shown in Fig. 7 offers an ideal example of recurrent bursts of energetic protons, which at the same time demonstrates that the extent of the electron source may be significantly smaller than that of the proton source. The extent of the source is of critical importance because a spatial restriction may lessen the chances for the electrons to be transported and detected downstream in magnetosheath. GEOTAIL was located at $(\mathrm{X}, \mathrm{Y}, \mathrm{Z})_{\mathrm{GSE}}=(-37.5,27.4,-6.2) R_{E}$. We note in particular the following:

1. The electrons display two distinct exodus channels of an estimated width of $\sim 6 R_{E}$ (derived by the local plasma velocity and burst duration), whereas the protons display five clearly distinct channels and the two major fluxes occurring simultaneously with the electron bursts. The ions of the CNO group essentially do not demonstrate any clear channel structure.

2. The five marked bursts of protons occurred with an equal number of excursions along the $\vartheta$ or $-B_{z}$ traces. The $\vartheta$ excursions towards zero are not accompanied by similar ones in the $\phi$ or the magnitude $B$ traces. Proton fluxes are observed when $\vartheta$ is less than $30^{\circ}$ and peaked with $\vartheta$ minima.

3. The average angular distribution for the energetic electron bursts "K3" and "K4", on the ecliptic plane, is shown in Fig. 8. The projected vector of the magnetic field on the ecliptic plane is also included. The almost undisturbed field deviates about $-27^{\circ}$ from the $\mathrm{X}$-axis. The constructed distribution concerns the $>110 \mathrm{keV}$ energetic electrons because only this electron channel provides measurements in 16 sectors. This distribution is typical for all the energetic particles, as well. The second angular distribution of Fig. 8 depicts the $7.5-230 \mathrm{keV}$ energetic protons from the EPIC/STICS sensor on the XZ plane. The EPIC/STICS sensor provides measurements in six equal $26.7^{\circ}$ polar sectors (from $+80^{\circ}$ to $-80^{\circ}$ ). The overall inferred result is that the energetic 
a Day $=16,1995$. Electrons $>38 \mathrm{keV}$. $01: 30: 43$ to $01: 31: 1$ UT.

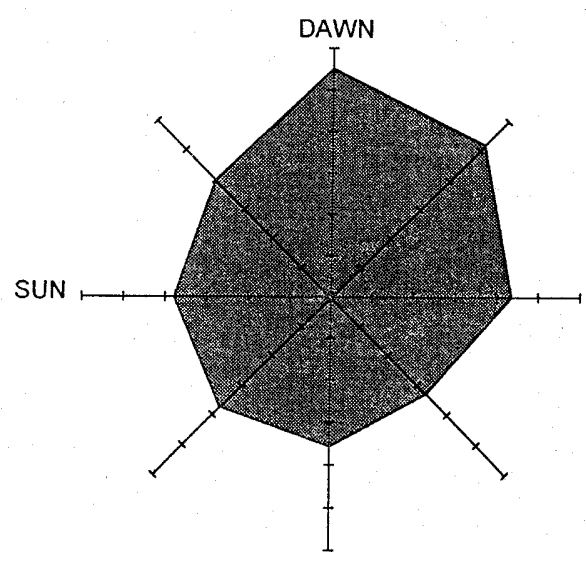

c Day $=16,1995$. CNO group $187-222 \mathrm{keV}$ $01: 33$ to $01: 35$ UT

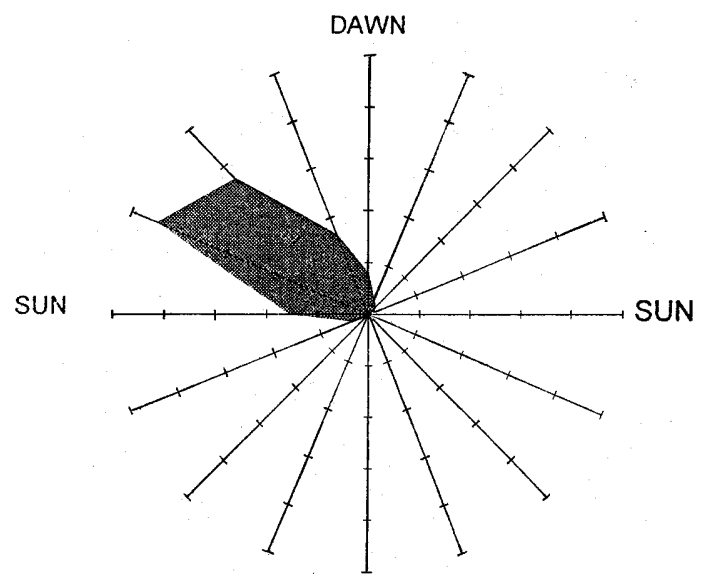

b

Day $=16,1995$

Protons $58-77 \mathrm{keV}$.

01:31:16 to $01: 32: 28$ UT.

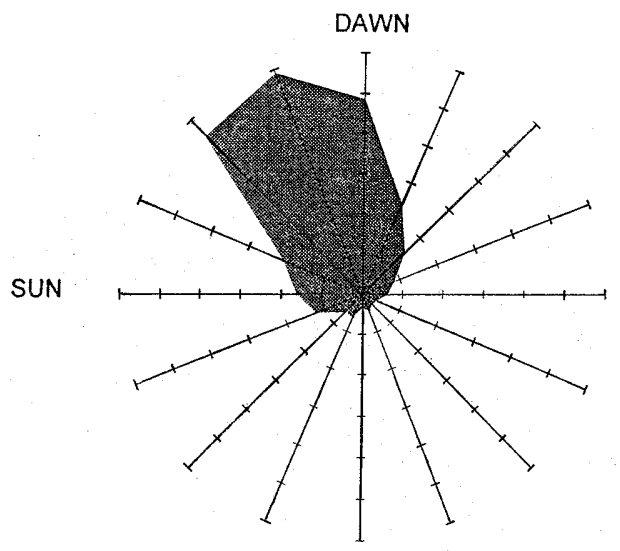

d

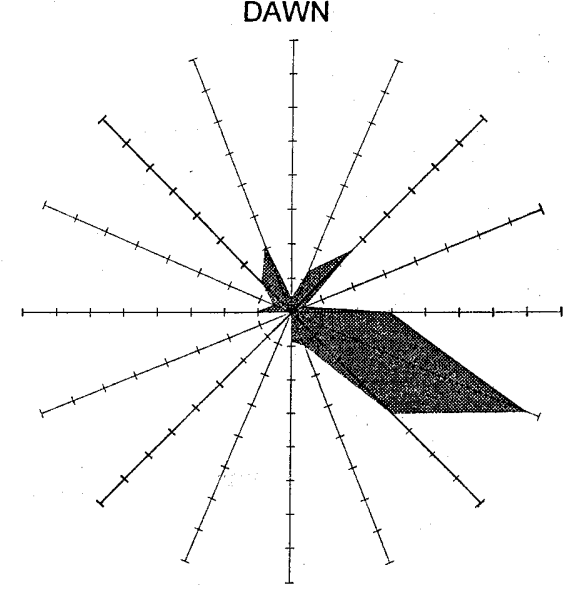

Fig. 6. a, $\mathbf{b}$ and $\mathbf{c}$ average angular distributions over the ecliptic plane XY correspond to the three peak fluxes of different energetic particle species presented in Fig. 5. The energetic electrons and protons are directed duskward along the local magnetic field direction. The angular distribution d displays an Earthward flux (in magnetosheath) resulting from an intensity gradient of energetic protons toward the equatorial plane protons are essentially concentrated around the ecliptic plane and stream tailward along the magnetic field lines.

An interpretation of the observed differentiation between energetic proton and electron fluxes may be as follows: The electron source is limited to lower latitudes of the magnetopause, and therefore, it is only when $B_{z}=0 \mathrm{nT}$ that the draping lines over the postnoon magnetopause thread through the GEOTAIL site. An alternative interpretation may be supported assuming a wavy magnetopause surface. That is, the five channels may correspond to equal approaches of the magnetopause to the spacecraft position. The differing gyro-radii of electrons and protons would explain very well why both species are not always observed simultaneously. A drawback in this consideration is that the streaming protons must be observed prior to and after the streaming electrons, which is not supported by the observations. Moreover, the latter interpretation cannot account for the occurrence of variations along the $B_{z}$ or $\vartheta$ trace in close association with the bursts.

\subsection{The magnetosheath region adjacent to the magnetopause}

At the magnetopause neighbourhood the magnetosheath energetic particles demonstrate a dramatically different behaviour compared to that exhibited in the evanescent exodus channels. During the time interval 00-08 UT of day 18, 1995, the spacecraft terminates its duskside magnetosheath traverse and repeatedly crosses the magnetopause. Figure 9 shows the three components of the GEOTAIL magnetic field (top three panels) concurrently with the energetic $58-77 \mathrm{keV}$ protons (fourth panel), 187-222 keV CNO ions (fifth panel) and $>38 \mathrm{keV}$ electrons (sixth panel). Before the final entry (at $\sim 07: 40$ UT) a few brief intervals of plasma sheet are encountered and shown by the negative (about $-20 \mathrm{nT}$ ) excursions of the $B_{x}$ component of the magnetic field. Figure 9 clearly demonstrates that the detection of magnetosheath energetic particles in the neighbourhood of the LLBL is not dependent on the condition $B_{z} \cong 0 \mathrm{nT}$. Intense particle fluxes on the 


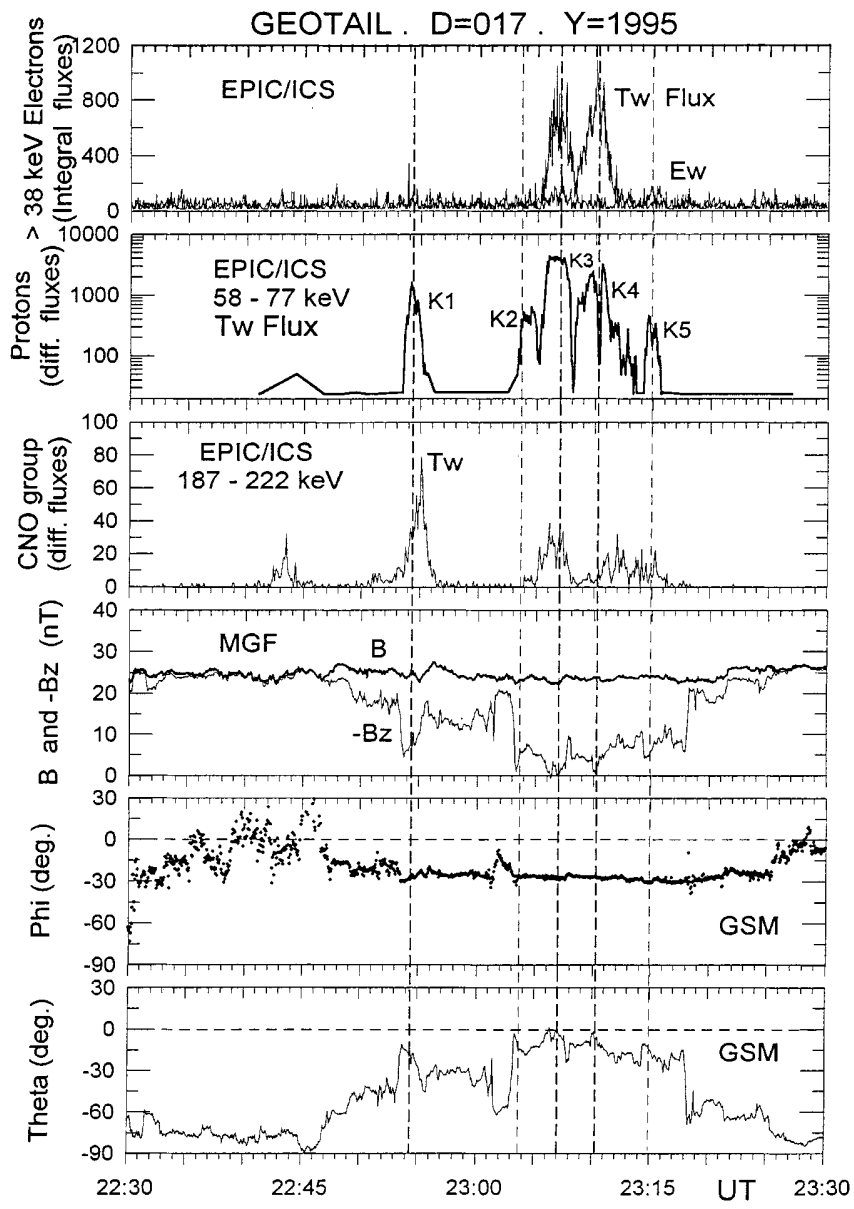

Fig. 7. From top to bottom the energetic $>38 \mathrm{keV}$ electrons, 58 $77 \mathrm{keV}$ protons (second panel), 187-222 keV CNO ions (third panel), the magnetic field magnitude and the $-B_{z}$ component (fourth panel), the azimuth (fifth panel) and the latitude (sixth panel) angles of the magnetic field are shown. Five distinct bursts of energetic protons that occurred in association with $B_{z} \cong 0 \mathrm{nT}$ are marked with the letter $K$

ecliptic plane have been recorded (for instance from 05:55 to 06:50 UT ), whereas the component $B_{z}$ is by far the dominant one and under such geometry no fluxes normally could be anticipated.

On the basis of these measurements, it could be inferred that a permanent layer of energetic particles always exists just outside the magnetopause (at least under the present magnetic field geometry). Throughout this layer the heavy ions flux level displays almost a "plateau". The angular distributions inside the layer are distinctly differentiated from the typical ones observed in the exodus channels (e.g. those in Fig. 8). The distributions here display the following features: (a) a profound symmetry of the tailward streaming protons around the $\mathrm{X}$-axis on the ecliptic plane (see Fig. 10a as a typical example); (b) the energetic protons stream tailward almost perpendicular to the dominant $B_{z}$ component of the local magnetic field and (c) the major fluxes of the 7.5-230 keV energetic protons (Fig. 10b) are concentrated on the ecliptic plane with a small southward flux along the magnetic field.

It seems that the draped magnetosheath magnetic field lines around the magnetosphere confine the escap-
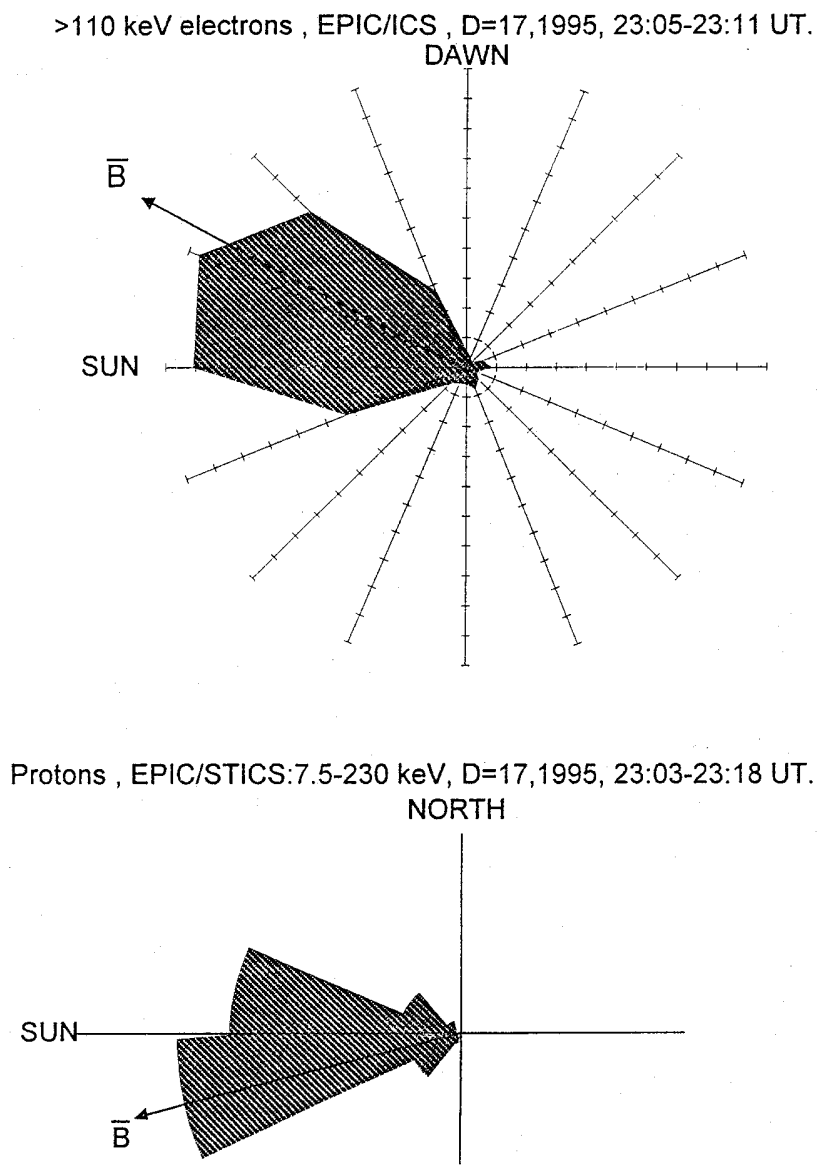

Protons, EPIC/ICS:228-342 keV, $D=17,1995,23: 05-23: 11$ UT

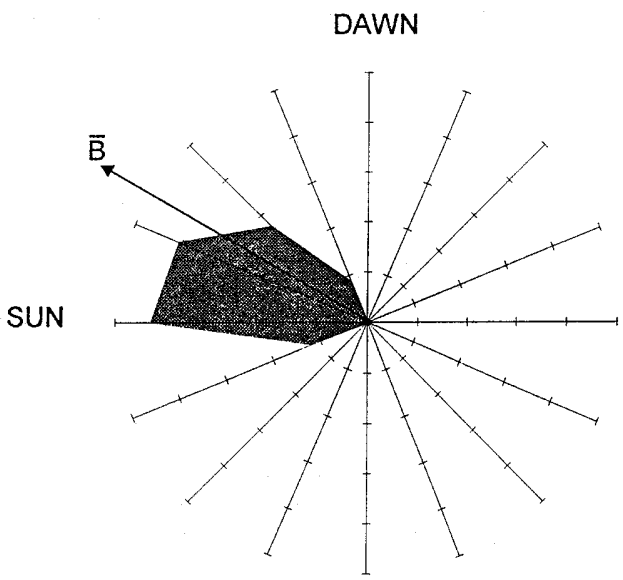

Fig. 8. Average angular distributions of energetic particles associated with the peak fluxes of Fig. 7. The first (of $>110 \mathrm{keV}$ electrons) and third distribution (of $228-343 \mathrm{keV}$ protons) visualize the field aligned character of streaming particles over the ecliptic plane, while the second distribution shows the fluxes of $7.5-230 \mathrm{keV}$ protons over the noon-midnight $\mathrm{XZ}_{\mathrm{GSE}}$ meridian plane demonstrating that the fluxes are mainly concentrated on the ecliptic plane. For the presented interval over the XY plane the $\varphi$ angle of field remained almost constant

ing particles to a narrow layer near the magnetopause. In this context, some energetic particles with a prevailing component of velocity along the magnetic field lines will 


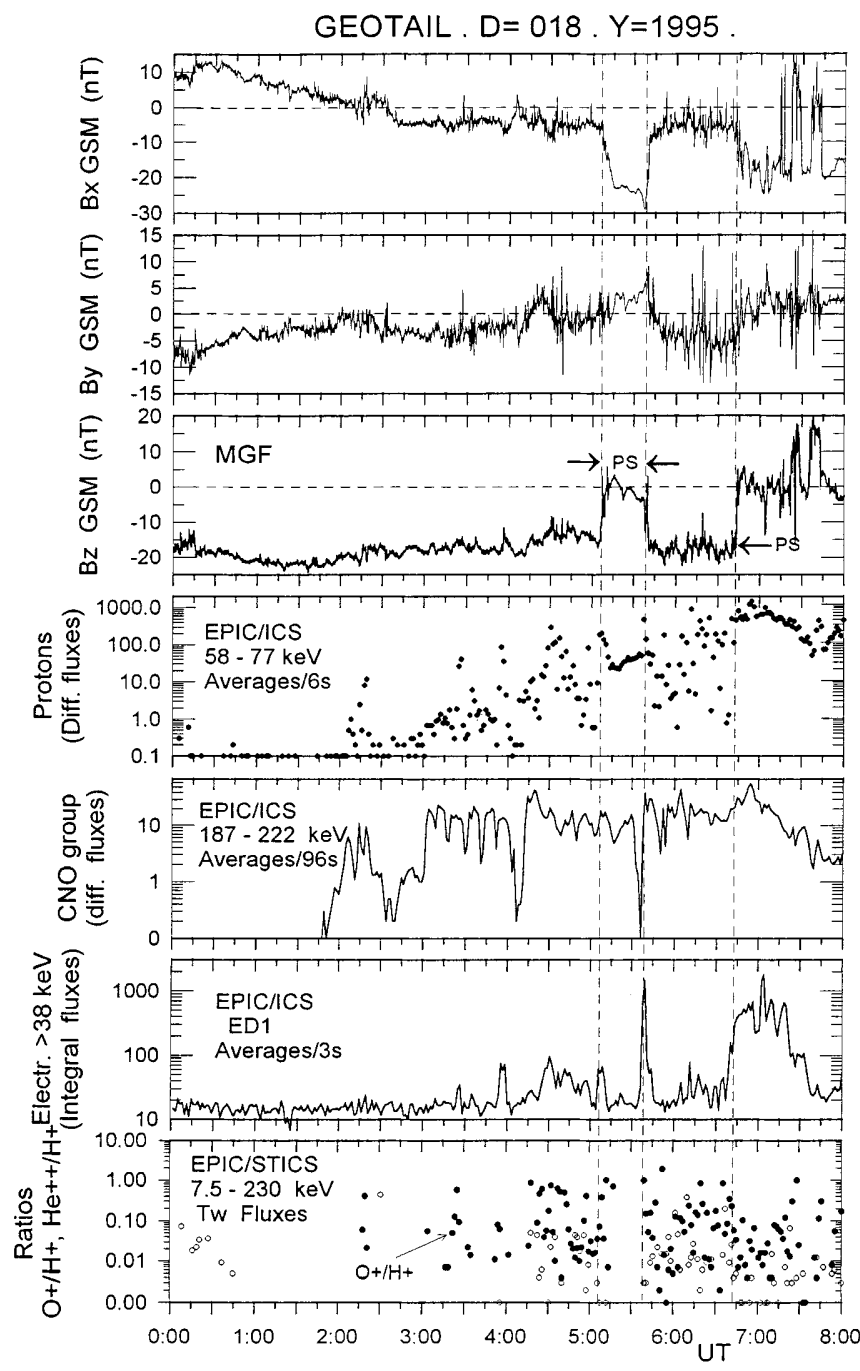

Fig. 9. During this interval from 00 to 08 UT on day 18, 1995, the spacecraft approaches and eventually repeatedly crosses the magnetopause boundary. The magnetotail domain is better diagnosed via the large negative excursions along the $B_{x}$ trace. Three indicative crossings occurred at $\sim 05: 06,05: 37$ and 06:36 UT. Fluxes of energetic particles adjacent to the magnetopause magnetosheath are detected with prevailing negative $B_{z}$ component suggesting the existence of a permanent layer just outside the LLBL region. The bottom panel shows the ratios of $\mathrm{O}^{+} / \mathrm{H}^{+}$and $\mathrm{He}^{++} / \mathrm{H}^{+}$

be quickly lost northward or southward. The rest of particles are almost trapped on field lines convecting downstream. The permanent layer particles may be energized via a merging mode because the local magnetosheath magnetic field is strongly southward. In the past, it has been suggested that a permanent layer of energetic electrons is present just outside the dayside (Meng and Anderson, 1970, 1975) and nightside magnetopause (Baker and Stone, 1978). Meng et al. (1981) found that, at the duskside magnetosheath, the energetic electrons are essentially restricted to a narrow band adjacent to the LLBL region, whereas the energetic protons are very extended around the high latitudes of magnetotail.

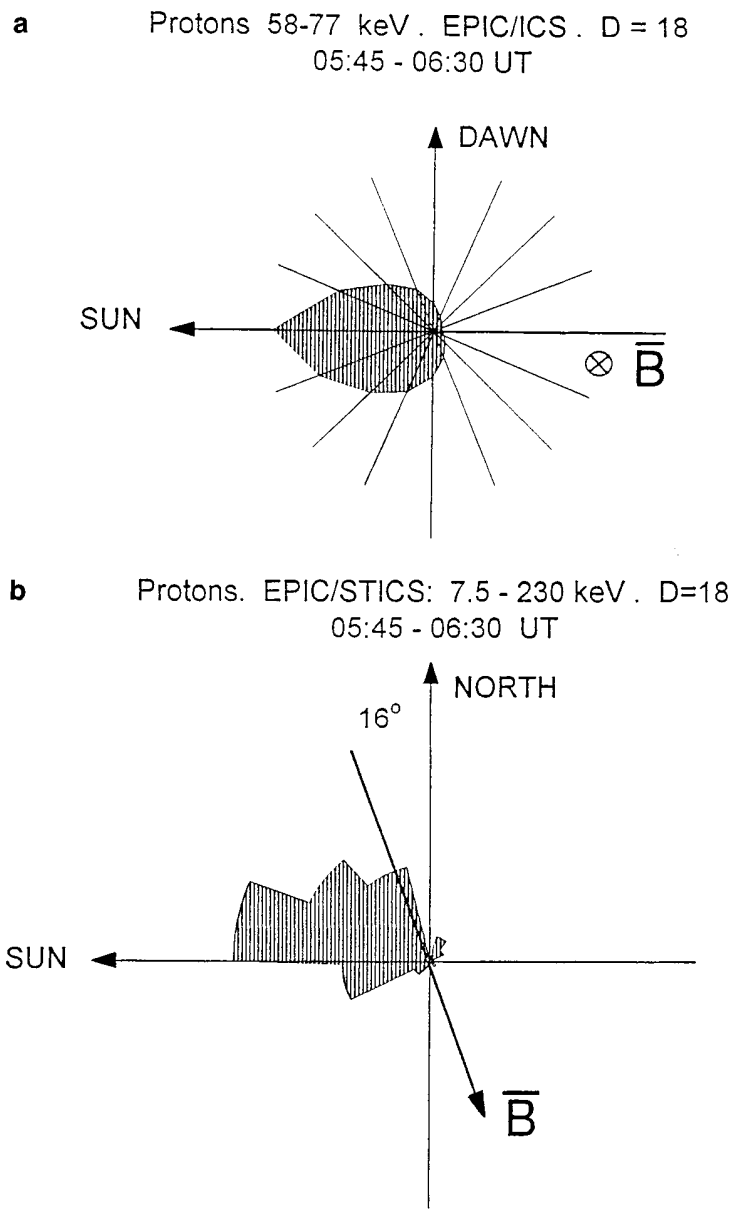

Fig. 10a, b. Two average angular distributions in format similar to that of Fig. 8 demonstrating a different behaviour as the magnetopause boundary is approached. On the $\mathrm{XY}_{\mathrm{GSE}}$ plane the $58-77 \mathrm{keV}$ protons convect tailward and almost perpendicular to the local magnetic field. On the $\mathrm{XZ}_{\mathrm{GSE}}$ plane the vector magnetic field deviates $\sim 16^{\circ}$ from the southward direction and although the dominant flux is the tailward one on the ecliptic plane, a minor southward flux along the magnetic field is also observable

\subsection{Energetic $\mathrm{O}^{+}$and $\mathrm{H}^{+}$ions display similar time profiles}

In the past, in an exceptional case with very active geomagnetic conditions, a brief burst $(\sim 20 \mathrm{~min})$ of energetic $\mathrm{O}^{+}$ions was observed streaming predominantly into the Sunward direction upstream of the Earth's bow shock (Möbius et al., 1986; Krimigis et al., 1986). Also, singly ionized suprathermal $(\mathrm{E}<17 \mathrm{keV} / \mathrm{q})$ oxygen is found, at least occasionally, to be present in the sub-solar magnetosheath (Peterson et al., 1982). In this work, intense fluxes of energetic $\mathrm{O}^{+}$ ions at the nightside magnetosheath are commonly observed. Moreover, a time coincidence between the observed major bursts of $\mathrm{O}^{+}$and $\mathrm{H}^{+}$ions can be clearly seen. Figure 11 shows the $58-77 \mathrm{keV}$ energetic proton fluxes in parallel with the $7.5-230 \mathrm{keV}$ singly ionized oxygens for an interval of $14 \mathrm{~h}$ of day 17, 1995. Singly ionized oxygen $\mathrm{O}^{+}$ions are of ionospheric origin. The observation of similar time-intensity profiles is of critical 

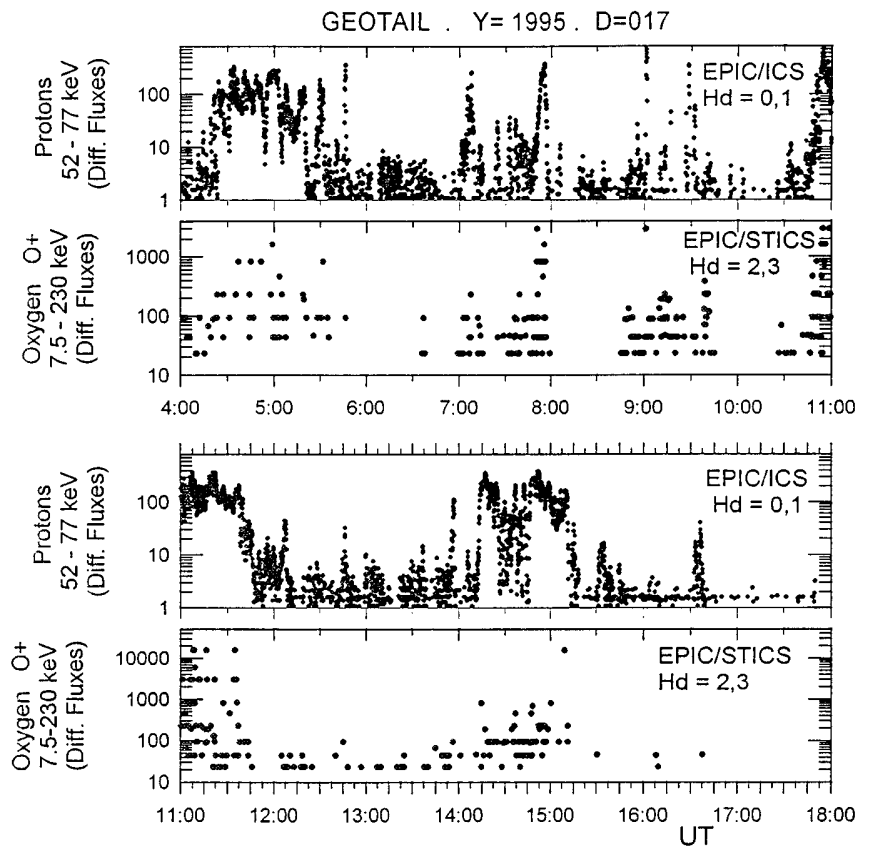

Fig. 11. Differential fluxes of 52-77 keV energetic protons and 7.5$230 \mathrm{keV}$ energetic $\mathrm{O}^{+}$ions during an interval of $14 \mathrm{~h}$ of day 17,1995 , when the $D_{s t}$ index continuously decreases. The magnetospheric origin ions as well as the energetic protons display almost similar time profiles suggesting their common source

importance leading us to consider that both $\mathrm{O}^{+}$and $\mathrm{H}^{+}$ species have the same origin.
The $\mathrm{O}^{+} / \mathrm{H}^{+}$ratio in the bottom panel of Fig. 9 (solid circles) of the $7.5-230 \mathrm{keV}$ tailward streaming protons and oxygen ions shows that, at times, the $\mathrm{O}^{+}$ions are as abundant as the protons at the nightside magnetosheath. The ratio of $\mathrm{O}^{+} / \mathrm{H}^{+}$is at least one order of magnitude higher than the ratio of $\mathrm{He}^{++} / \mathrm{H}^{+}$presented in the same panel (open circles). The $\mathrm{He}^{++}$ions are of solar origin. At the plasma sheet the ratio $\mathrm{He}^{++} / \mathrm{H}^{+}$ decreases when the geomagnetic activity level increases, whereas the ratio of $\mathrm{O}^{+} / \mathrm{H}^{+}$responds to the opposite direction (Sharp et al., 1982). In conclusion, the similarity of the magnetosheath oxygen and proton time profiles along with the increased ratio of $\mathrm{O}^{+} / \mathrm{H}^{+}$, as it is the case with increased activity inside the magnetosphere, support the magnetospheric origin of both ions in the exodus channels.

\subsection{Magnetosheath spectra}

In the following we present two energy spectra corresponding first to the region adjacent to the magnetopause permanent layer and the other to a wide exodus channel. The particle spectra provide some clear indications for certain aspects of the magnetospheric dynamics and particle properties determining their origin, energization mechanisms, transport paths etc. In Fig. 12a the open and solid circle symbols give the spectra displaying the relative energetic proton abundances both in the plasma sheet and the magnetosheath. The magnetosheath spectrum is the overall response within the intervals 02:00-05:00 UT and 05:45-06:30

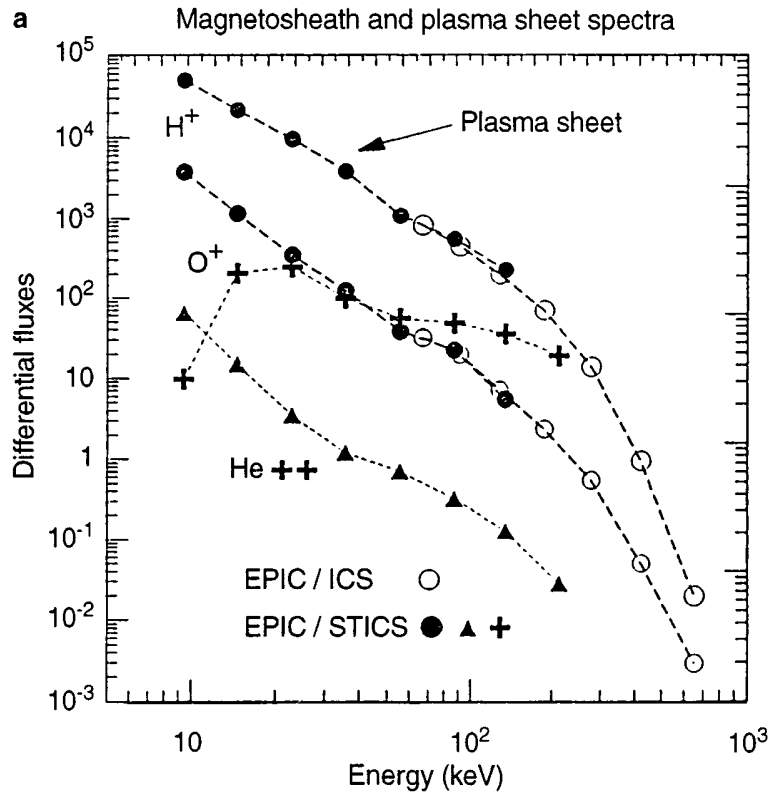

Fig. 12. a Magnetosheath (average response for intervals 02:00-05:00 UT and 05:45-06:30 UT of day 18, 1995) and plasma sheet (average response from 06:50 to 07:10 UT of the same day) proton spectra showing almost similar spectral shape and a gradient of energetic particles toward the magnetopause. The open (solid) symbols correspond to the ICS (STICS) detector of the EPIC experiment. The singly ionized oxygens (crosses) and $\mathrm{He}^{++}$ions (triangles)

b

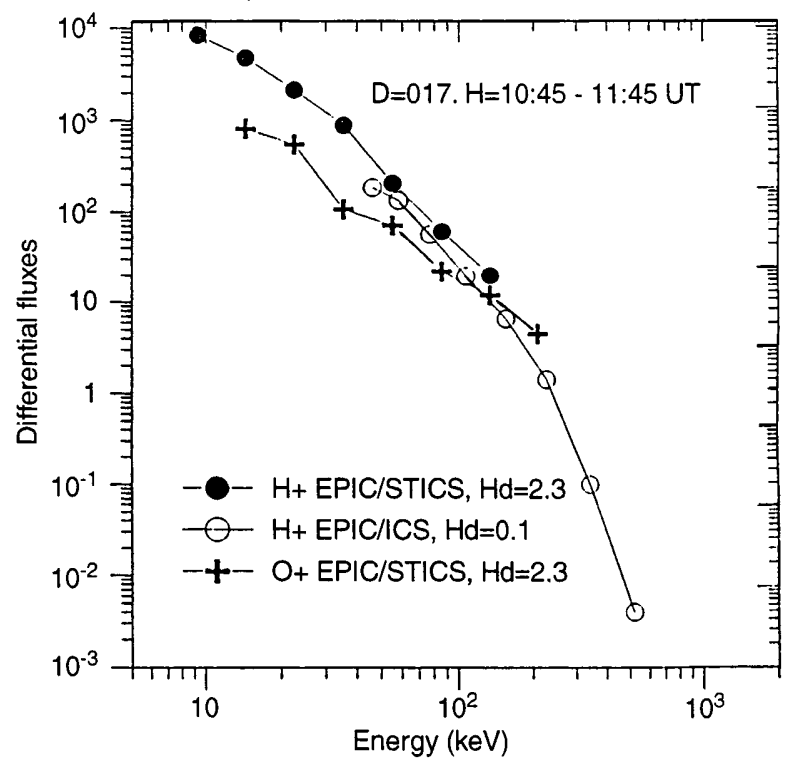

correspond to the mentioned magnetosheath period. The $\mathrm{O}^{+}$ions are detected with as low as $\sim 10 \mathrm{keV}$ energies and for the energy range 20 $60 \mathrm{keV}$ are of equal abundance with the protons. b Magnetosheath spectra corresponding to an exodus channel interval from 10:45 to 11:45 UT of day 17, 1995. The symbols are similar to those of a. In this case the magnetosheath is flooded with magnetospheric origin ions reaching a lowest energy of $\sim 15 \mathrm{keV}$ for oxygens 
UT, while that of the plasma sheet is the average spectrum for 06:50-07:10 UT of the same day 18, 1995. The almost similar spectral shape, as well as the fact that the gradient of energetic particles is directed toward the magnetopause probably are manifestations of their magnetospheric origin. The plasma sheet may be an adequate source of the magnetosheath population. Certainly, between the two spectra there is a time progression. In Fig. 12a the spectra of magnetosheath $\mathrm{O}^{+}$and $\mathrm{He}^{++}$ions are added. Additional notable observations are as follows:

1. "Tracer" ions of ionospheric-magnetospheric origin are detected inside the magnetosheath with energies as low as $\sim 9.5 \mathrm{keV}$.

2. The fluxes of solar origin $\mathrm{He}^{++}$ions are much lower than those of $\mathrm{O}^{+}$or $\mathrm{H}^{+}$. At $\sim 30 \mathrm{keV}$ the percentage of $\mathrm{H}^{+} / \mathrm{He}^{++}$is about $1 \%$.

3. At energies ranging from 20 to $60 \mathrm{keV}$ the $\mathrm{H}^{+}$and $\mathrm{O}^{+}$ions have nearly the same abundances. In general, the proton spectrum is softer than the $\mathrm{O}^{+}$one.

The spectra in Fig. 12a correspond to the region adjacent to the magnetopause. A different point of view is given via the spectra of Fig. 12b, corresponding to a broad exodus channel from 10:45 to 11:45 UT of day 17, 1995. A prominent inference is that the ionosphere $\mathrm{O}^{+}$ ions are channelled (similarly to the protons) away from the magnetopause at all energies above $\sim 15 \mathrm{keV}$. This seems to agree with Paschalidis et al. (1994), who have found significant magnetospheric contribution in the dayside magnetosheath reaching energies as low as $\sim 10 \mathrm{keV}$. At this lower threshold, the ratio of $\mathrm{O}^{+} / \mathrm{H}^{+}$is $\sim 0.17$. It is evident that the high energy oxygen ions have a higher chance to escape outside the magnetosphere and be transported away.

\section{Discussion}

We have examined a duskside magnetosheath traversal by the GEOTAIL spacecraft from about $\mathrm{X}=-15$ to $-40 R_{E}$. We are interested in the issues of morphology and properties of the "exodus channels". The origin of the outward channelled magnetosheath energetic particles is an outstanding problem, too. The detection of $\mathrm{O}^{+}$ ions in magnetosheath is not something new by itself. Similarly, spectra constructed in the magnetosheath could be found in past works. In this all these are used as tools and serve for a better understanding of the phenomenon of the exodus of magnetospheric particles. Again, we point out that the large majority of magnetosheath works are restricted to the dayside rather than the nightside region. A fundamental feature of the observed particle exodus is that it occurred simultaneously with the evolution of a great storm leading to high abundance of ionospheric ions inside the magnetosphere (see Lennartsson and Sharp, 1982; Daglis, 1997), and the following release of $\mathrm{O}^{+}$ions outward in the magnetosheath. Therefore, these tracer ions provide good evidence for the path through which the dominant ions have been transported to the spacecraft.
Away from the magnetopause, the energetic proton fluxes are switched on or off depending essentially upon the condition that the magnetic field remains on or out of the $B_{z} \cong 0 \mathrm{nT}$ plane. The in situ evidence justifying the key-role of this condition could be as follows: first, the systematic and recurrent detection of fluxes each time the $B_{z}$ component of the magnetic field approaches zero (irrespectively of the recorded $B_{x}$ and $B_{y}$ components) as in Fig. 4. Second, the magnitude and azimuthal angle of the magnetic field do not change in many of the observed fluxes (see Figs. 4 and 7). And third, the fact that the energetic proton fluxes are highly sensitive even to micro-excursions of the $B_{z}$ component ( $B_{z}$ or $\vartheta$ trace).

The role of the $B_{z}$ component in controlling the escaping magnetospheric particles is understood as follows: the magnetic field lines drape the dayside magnetosphere at low latitudes and are loaded with the escaping magnetospheric population. These particles stream along the magnetic field lines and are subsequently measured by the EPIC sensors. Equatorial magnetosheath magnetic field lines typically make their closest approach to the magnetosphere at local times near 1500 LT (see Sibeck et al., 1987a). If the $B_{z}$ component is significant then the streaming particles will be quickly displaced well above or below the ecliptic plane, failing to encounter the GEOTAIL detector almost on the ecliptic plane. Hence, the $B_{z}$ component is the most critical parameter which favours or precludes whether the energetic particles are channelled far away downstream. On this basis it is conceivable that: (a) the IMF largely modulates the space and dictates the suitable condition under which the transport and detection of particles away from the magnetopause is possible; (b) the condition $B_{z} \cong 0 \mathrm{nT}$ establishes a magnetic connection between the spacecraft and the magnetosphere; and (c) the magnetosphere as the supplying source needs to be filled up with energetic particles in order to provide them.

It was reported (West and Buck, 1976) that magnetosheath fluxes occurred with depressed and turbulent magnetic field. Instead, in this work intense bursts are observed even with almost unperturbed magnetic field magnitudes (see the bursts of Figs. 4 and 7). The turbulent character may contribute to the particle dispersion and propagation, but the most important factor remains the magnetic field orientation.

An interpretation of the observed dispersion structure in peak fluxes of Fig. 5 may be as follows: as the vector magnetic field scans progressively all the directions from northward to southward lying at the same time on the YZ plane, the magnetic field lines may be connected with the sources of the energetic particles. The energetic electrons peak precedes that of the protons and therefore the electrons source must be displaced slightly more northward than the protons one. Most probably, the electrons follow the trajectories along the draping magnetic field lines outside the magnetopause over the YZ cross-sectional plane of the magnetotail. The magnetospheric energetic electrons $(\mathrm{E}>25 \mathrm{keV})$ drift eastward, hit the pre-noon magnetopause and leak out (Anagnostopoulos et al., 1986; 
Sibeck et al., 1987b; Sibeck and McEntire, 1988). The latter statement is also supported by the fact that the majority of energetic proton (electron) intense flux events are found on the dusk (dawn) magnetotailmagnetosheath (see Meng et al., 1981; Krimigis and Sarris, 1979). The escaping electrons in the dawn magnetosheath via the draped magnetic filed lines over the magnetopause are transported to the GEOTAIL site. Conversely, the magnetospheric energetic protons are considered to leak out from the lower latitudes of the dusk magnetopause. Therefore the dispersion between protons and electrons is considered to be a spatial effect. The energetic heavy $\mathrm{CNO}$ ions and protons have the same source. The best contact with the ions source must be anticipated with zero latitude angle of the magnetic field. The observed delay ( $\sim 70 \mathrm{~s})$ between the peak fluxes of the CNO ions and protons is considered to be a temporal effect corresponding to the different travel times. The faster protons arrived earlier than the $\mathrm{CNO}$ ions. In addition, the magnetic field direction was changing gradually and this causes a further lengthening of travelling path for the CNO ions (and therefore a further increase of the needed travel time).

This interpretation is further supported by an additional distinctive point: the proton fluxes prior to the peak display a Sunward flow character. The angular distribution of Fig. 6d demonstrates such a situation. And this feature may be a genuine result of a $\mathbf{B X} \nabla \mathbf{U}$ anisotropy due to a large north-south gradient in the proton intensities (see Sarafopoulos and Sarris, 1991), since the plasma sheet energetic particles are expected to escape outside the magnetopause primarily in the vicinity of the $Z_{\mathrm{GSM}}=0$ plane and the GEOTAIL is located to the north of that plane, at $(\mathrm{X}, \mathrm{Y}$, $\mathrm{Z})_{\mathrm{GSM}}=(-15.5,28.5,6.6) R_{E}$. This time the magnetic field points eastward.

The claim about the magnetospheric origin of energetic particles in exodus channels could be supported by the following arguments:

1. The detected major magnetosheath fluxes of streaming protons along the magnetic field lines display the same time profile as that of $\mathrm{O}^{+}$ions. In addition, the plasma sheet population of energetic particles alone suffices to warrant the lower observable fluxes in magnetosheath. The density gradient of energetic particles is directed toward the magnetopause (Williams et al., 1988; Traver et al., 1991; Paschalidis et al., 1994; Eastman and Christon, 1995).

2. The "exodus channel" emerges each time the extrapolated local magnetic field lines are draped around the lower magnetotail latitudes. In one case study, at the dayside magnetosheath, a similar observation is referred to by Sibeck et al. (1987a): the maximum flux was related to the equatorial region of greatest particle escape.

3 . It could be argued that the energetic population streaming along an "exodus channel" could be energized via the shock drift acceleration (SDA) mechanism. In this case the shock drift accelerated protons at $\mathrm{E}_{\mathrm{p}} \geq 290 \mathrm{keV}$ and then transmitted downstream must present a double-peaked anisotropy nearly perpendicu- lar to the magnetic field (see Anagnostopoulos and Kaliabetsos, 1994). In the present work, we failed to observe such a characteristic signature as the fingerprint of the SDA mechanism. For example the average angular distribution for the EPIC/ICS proton channel P6 (i.e. $228 \leq \mathrm{E}_{\mathrm{p}} \leq 342 \mathrm{keV}$ ) corresponding to the two exodus channels "K3" and "K4" of Fig. 7 is shown in Fig. 8. Instead of a double-peaked angular distribution, a profound field aligned anisotropy is seen.

4. The Fermi acceleration is widely considered as another candidate mechanism working over quasi-parallel bow shocks and producing energetic particles, which in turn, could be swept downstream in the magnetosheath (Scholer et al., 1980; Fuselier, 1994). Our prominent examples of exodus channels, like those shown in Fig. 7, took place at the dusk-side magnetosheath, although the typical "Archemedian spiral" angle of the IMF would favour the Fermi acceleration over the dawnside part of the bow shock. In addition, the Fermi acceleration mechanism is unable to produce energetic electrons, like those observed in exodus channels.

In Fig. 12 it is shown that the magnetosheath and magnetospheric spectra resemble each other greatly, and therefore one must conclude that escaping magnetospheric particles dominate any population of magnetosheath particles energized by merging. But the in situ measurements show something more against the merging process: first, in the presented case study of Fig. 5 the burst occurred with $B_{z} \cong 0 \mathrm{nT}$ and dominating the $B_{y}$ component. Thus the lines drape around the magnetopause over the $\mathrm{YZ}$ plane and our interpretation of the observed dispersion structure for different species is based on the notion of leakage. We think that the nonmerging mode model is able to produce such a structure. Second, in most cases (see Figs. 4 and 7), however, the bursts occurred with $B_{z} \cong 0$ and dominating the $B_{x}$ component. In this magnetic field geometry the magnetosheath extrapolated lines are draped at the post-noon magnetopause perpendicularly to the local magnetosphere magnetic field. Therefore, the merging mechanism is also unlikely to work. So a much better explanation is that the particles are leaking out all the time and that when the magnetic field connects the spacecraft to the tail, they stream away and are observed at the spacecraft.

Scholer et al. (1984), in one case study at the dawn side of the distant magnetosheath $\left(\sim 50 R_{E}\right.$ downstream) observed energetic electrons and protons occurring almost simultaneously in bursts of $\sim 2$ min duration. These particles are considered streaming along Earthrooted magnetic field lines (that is, as remnants of flux transfer events). In this work the magnetosheath particle bursts are scrutinized further. An objection in respect to this interpretation, and in the light of our observations, is that the reconnection process must work continuously over the magnetopause and the resulting streaming particles are seen whenever $B_{z} \cong 0$.

Anagnostopoulos et al. (1986) in their Fig. 5 show magnetospheric ions on outer dusk magnetosheath magnetic field lines which do not encounter the magne- 
topause. This may understood as follows: first, this figure is a sketch drawn mainly on the base of measurements of escaping particles upstream of the bow shock. Second, a major implication of the figure seems to be a steep dawnward gradient of energetic ions in the dusk magnetosheath, which is largely unproved at present. Third, they have used $5.5 \mathrm{~min}$ averages of energetic particles, whereas the duration of our detailed studied channels usually are less than 5 min. Fourth, we have assumed that the typical duskside magnetosheath magnetic field lines drape the post-noon magnetopause. In the present work streaming particles are commonly observed, except from the case of electrons detected when the $B_{y}$ component of the magnetic field was dominant (see Fig. 5).

It is worth noticing that a paradoxical result that might be reached by hypothetical statistical surveys in the magnetosheath. Because the fluxes of energetic particles are strongly dependent on the magnetic field direction, it follows that during intervals of great storms when the $B_{z}$-negative component of the magnetic field clearly dominates, no fluxes could be detected wellinside the magnetosheath. Conversely, during long periods of lower geomagnetic activity and suitable geometry of the magnetosheath magnetic field persistent fluxes could occur.

Whenever the magnetospheric particles escape, the magnetosheath magnetic field guides them toward the bow shock. Those particles that succeed in traversing the magnetosheath to the bow shock provide a source of particles for upstream events. (Sarris et al., 1976; Scholer et al., 1981; Anagnostopoulos et al., 1986; Sarris et al., 1987; Sibeck et al., 1987b; Baker et al., 1988).

\section{Synopsis}

Our observations concerning the origin and properties of energetic particles in the duskside magnetosheath lead us to the following conclusions: (a) the bursty character of the fluxes depends mainly from the $B_{z}$ component orientation (which, in turn, is probably dictated by the solar wind magnetic field topology; (b) the magnetospheric origin of energetic particles is established on the basis of spectra and $\mathrm{O}^{+}$fluxes; (c) the observation of a dispersion structure occurring under a distinct magnetic field geometry supports the leakage process against the merging one for the origin of energetic particles in the magnetosheath; (d) Sunward magnetosheath detected fluxes provide evidence for the presence of a gradient of energetic protons toward the $\mathrm{Z}_{\mathrm{GSM}}=0$ plane; and (e) in general, the energetic protons stream along the magnetic field lines, except in the layer adjacent to the magnetopause, where they merely convect perpendicular to the local field.

Acknowledgements. We are grateful to Prof. L.A. Frank and Dr. $\mathrm{K}$. Ackerson for providing high resolution data from the CPI/SWA instrument. Also, we thank T. Kamei and M. Sugiura for providing the Dst data, Dr. D. Sibeck for helpful conversations, as well as both of the referees for their constructive comments. This work was supported by the contract PENED95/878 of the GSRT (General Secretariat of Research and Technology, Greek Ministry for Development).

Topical Editor K.-H. Glassmeier thanks J. Woch and another referee for their help in evaluating this paper.

\section{References}

Anagnostopoulos, G. C., and G. D. Kaliabetsos, Shock drift acceleration of energetic $(\mathrm{E} \geq 50 \mathrm{keV})$ protons and $(\mathrm{E} \geq 37 \mathrm{keV} / \mathrm{n})$ alpha particles at the Earth's bow shock as a source of the magnetosheath energetic ion events, J. Geophys. Res., 99, 2335-2349, 1994.

Anagnostopoulos, G. C., E. T. Sarris, and S. M. Krimigis, Magnetospheric origin of energetic $(\mathrm{E} \geq 50 \mathrm{keV})$ ions upstream of the bow shock: the October 31, 1977 event, J. Geophys. Res., 91, 3020-3028, 1986.

Asbridge, J. R., S. J. Bame, J. T. Gosling, G. Paschmann, and N. Sckopke, Energetic plasma ions within the Earth's magnetosheath, Geophys. Res. Lett., 5, 953-955, 1978.

Baker, D. N., and E. C. Stone, The magnetopause energetic electron layer, 1. Observations along the distant magnetotail, J. Geophys. Res., 83, 4327-4338, 1978.

Baker, J. B., R. D. Belian, T. A. Fritz, P. R. Higbie, S. M. Krimigis, D. G. Sibeck, and R. D. Zwickl, Simultaneous energetic particle observations at geostationary upstream orbit and in the upstream solar wind: evidence for leakage during the magnetospheric compression event of November 1, 1984, J. Geophys. Res., 93, 14 317-14 327, 1988.

Eastman, T. E., and S. Christon, Ion composition and transport near the Earth's magnetopause, in Geophysical monograph 90, Physics of the Magnetopause, Eds. P. Song, B. U. Ö. Sonnerup, and M. F. Thomsen, 131-137, 1995.

Daglis, I., The role of magnetosphere-ionosphere coupling in magnetic storm dynamics, Magnetic storms, in Geophysical monograph 98 Eds. B. T. Tsurutani, W. D. Gonzalez, and Y. Arballo, 107-116, 1997.

Frank L. A., K. L. Ackerson, W. R. Paterson, J. A. Lee, M. R. English, and G. L. Pickett, The comprehensive plasma instrument (CPI) for the GEOTAIL spacecraft, J. Geomagn. Geoelectr., 46, 23-37, 1994.

Fuselier, S., A comparison of energetic ions in the plasma depletion layer and the quasi-parallel magnetosheath, J. Geophys. Res., 99, 5855-5868, 1994.

Hones, E. W., Jr., S.-I. Akasofu, S. J. Bame, and S. Singer, Outflow of plasma from the magnetotail into the magnetosheath, J. Geophys. Res., 77, 6888-6695, 1972.

Gonzalez, W. D., and B. T Tsurutani, Criteria of interplanetary parameters causing intense magnetic storms $\left(D_{s t}<-100 \mathrm{nT}\right)$, Planet. Space Sci., 35, 1101-1109, 1987.

Gonzalez, W. D., J. A. Joselyn, Y. Kamide, H. W. Kroehl, G. Rostoker, B. T. Tsurutani, and V. M. Vasyliunas, What is a geomagnetic storm?, J. Geophys. Res., 99, 5771-5792, 1994.

Kokubun, S., T. Yamamoto, M. H. Acuna, K. Hayashi, K. Shiokawa, and H. Kawano, The GEOTAIL magnetic field experiment, J. Geomagn. Geoelectr., 46, 7-21, 1994.

Krimigis, S., and E. T. Sarris, Energetic particle bursts in the Earth's magnetotail, in Dynamics of the magnetosphere, Eds. S.-I. Akasofu, 599-630, 1979.

Krimigis, S. M., D. G. Sibeck, and R. W. McEntire, Magnetospheric particle injection and the upstream ion event of September 5, 1984, Geophys. Res. Lett., 13, 1376-1379, 1986.

Lennartsson, W., and R. D. Sharp, A comparison of the 0.1$17 \mathrm{keV} / \mathrm{e}$ ion composition in the near equatorial magnetosphere between quiet and disturbed conditions, J. Geophys. Res., 87, 6109-6120, 1982.

Lepping, R. P., M. H. Acuna, L. F. Burlaga, W. M. Farrel, J. A. Slavin, K. H. Schatten, F. Mariani, N. F. Ness, F. M. Neubauer, Y. C. Whang, J. B. Byrnes, R. S. Kennon, P. V. Panetta, 
J. Scheifele, and E. M. Worley, The WIND magnetic filed investigation, Space Sci. Rev., 71, 207-229, 1995.

Meng, C.-I., and K. A. Anderson, A layer of energetic electrons $(40>\mathrm{keV})$ near the magnetopause, 75, 1827-1836, 1970.

Meng, C.-I., and K. A. Anderson, Characteristics of the magnetopause energetic electron layer, J. Geophys. Res., 80, 4237-4243, 1975.

Meng, C.-I., A. T. Y. Lui, S. M. Krimigis, and S. Ismail, Spatial distribution of energetic particles in the distant magnetotail, J. Geophys. Res., 86, 5682-5700, 1981.

Möbius, E., D. Hovestadt, B. Klecker, M. Scholer, F. M. Ipavich, C. W. Carlson, and R. P. Lin, A burst of energetic $\mathrm{O}^{+}$ions during an upstream particle event, Geophys. Res. Lett., 13, 1372-1375, 1986.

Paschalidis, N. P., E. T. Sarris, S. M. Krimigis, R. W. McEntire, M. D. Levine, I. A. Daglis, and G. C. Anagnostopoulos, Energetic ion distributions on both sides of Earth's magnetopause, J. Geophys. Res., 99, 8687-8703, 1994.

Peterson, W. K., E. G. Shelley, G. Haerendel, and G. Paschmann, Energetic ion composition in the subsolar magnetopause and boundary layer, J. Geophys. Res., 87, 2139-2145, 1982.

Sarafopoulos, D. V., and E. T. Sarris, Determination of the primary region of access of energetic particles from their source in the Earth's plasma sheet, Ann. Geophysicae, 9, 429-441, 1991.

Sarris, E. T., S. M. Krimigis, and T. P. Armstrong, Observations of magnetospheric bursts of high-energy protons and electrons at $\sim 35 \mathrm{R}_{\mathrm{E}}$ with Imp7, J. Geophys. Res., 81, 2341-2355, 1976.

Sarris, E. T., S. M. Krimigis, C. O. Bostrom, and T. P. Armstrong, Simultaneous multispacecraft observations of energetic proton bursts inside and outside the magnetosphere, J. Geophys. Res., 83, 4289-4305, 1978.

Sarris, E. T., G. C. Anagnostopoulos, and S. M. Krimigis, Simultaneous measurements of energetic ion $(\geq 50 \mathrm{keV})$ and electron ( $\geq 220 \mathrm{keV})$ activity upstream of Earth's bow shock and inside the plasma sheet; magnetospheric source for November 3 and December 3, 1977 upstream events, J. Geophys. Res., 92, 12 083-12 096, 1987.

Sibeck, D. G., R. W. McEntire, A. T. Y. Lui, R. E. Lopez, S. M. Krimigis, R. B. Decker, L. J. Zanetti, and T. A. Potemra,
Energetic magnetospheric ions at the magnetopause: leakage or merging?, J. Geophys. Res., 92, 12 097-12 114, 1987a.

Sibeck, D. G., R. W. McEntire, A. T .Y. Lui, S. M. Krimigis, L. J. Zanetti, and T. A. Potemra, The magnetosphere as a source of energetic magnetosheath ions, Geophys. Res. Lett., 14, 1011$1014,1987 \mathrm{~b}$

Sibeck, D.G., and R. W. McEntire, Multiple satellite observations of leakage of particles from the magnetosphere, Adv. Space Res., 8, 9.201-9.216, 1988.

Scholer, M., F. M. Ipavich, G. Gloeckler, and D. Hovestadt, Conditions for acceleration of energetic ions $\geq 30 \mathrm{keV}$ associated with the Earth's bow shock, J. Geophys. Res., 85, 4602-4606, 1980.

Scholer, M., D. Hovestadt, F. M. Ipavich, and G. Gloeckler, Upstream energetic ions and electrons: Bow shock-associated or magnetospheric origin?, J. Geophys. Res., 86, 9040-9046, 1981.

Scholer, M., D. Hovestadt, G. Gloeckler, B. Klecker, F. M. Ipavich, and R.D. Zwickl, Magnetospheric ions and electrons in the distant magnetosheath at $\sim 50 \quad R_{E}$ and $\sim 180 \quad R_{E}$ : ISEE-3 observations, Geophys. Res. Lett., 11, 1098-1101, 1984.

Sharp, R. D., W. Lennartsson, W. K. Peterson, and E. G. Shelley, The origins of the plasma in the distant plasma sheet, J. Geophys. Res., 87, 10 420-10 424, 1982.

Traver, D. P., D. G. Mitchell, D. J. Williams, L. A. Frank, and C. Y. Huang, Two encounters with the flank low-latitude boundary layer: Further evidence for closed field topology and investigation of the internal structure, J. Geophys. Res., 96, 21025 $21035,1991$.

West, H. I., and R. M. Buck, Observations of $>100 \mathrm{keV}$ protons in the Earth's magnetosheath, J. Geophys. Res., 81, 569-584, 1976.

Williams, D. J., Observations of significant magnetosheath antisolar energy flow, J. Geophys. Res., 84, 2105-2108, 1979.

Williams, D. J., D. G. Mitchell, L.A. Frank, and T. E. Eastman, Three-dimensional magnetosheath plasma ion distributions from $200 \mathrm{eV}$ to $2 \mathrm{Mev}$, J. Geophys. Res., 93, 12 783-12 794, 1988.

Williams, D. J., R. W. McEntire, C. Schemm II, A. T. Y. Lui, G. Gloeckler, S. P. Christon, and F. Gliem, GEOTAIL energetic particles and ion composition instrument, J. Geomagn. Geoelectr., 46, 39-57, 1994. 